\title{
Hepatocyte Lacks Insulin-Mediated Glucose Transporter Translocation Mechanism in Rat
}

\author{
Jong-Sik Hah \\ Department of Physiology, College of Medicine, Ewha Womans University \\ $=$ Abstract $=$
}

The subcellular distribution of glucose transporter in rat hepatocytes was studied in the absence and presence of insulin by measuring glucose-sensitive cytochalasin B binding sites and immunoreactivities to antibodies specific to hepatocyte glucose transporter. Total hepatocyte membranes bound cytochalasin $\mathrm{B}$ at a class of glucose-sensitive sites with a $\mathrm{K}_{\mathrm{d}}$ of $1.6 \times 10^{-6} \mathrm{M}$, and a $\mathrm{B}_{\mathrm{t}}$ of $6.8 \mathrm{pmol} / \mathrm{mg}$ protein. The glucose-sensitive cytochalasin $\mathrm{B}$ binding sites were found in various subcellular membrane fractions with a relative abundance of $47 \%$ in a plasma membrane-nuclei-mitochondria-enriched fraction(PM/NM), $29 \%$ in a lysosome-enriched fraction(LYSO), $16 \%$ in a Golgi-enriched, high density microsomal fraction (HDM) and $8 \%$ in the low density microsome fraction(LDM). Relative abundances of two well known plasma membrane markers, 5'-nucleotidase and cell surface carbohydrate label, on the other hand, were found to be $48 \sim 50 \%$ in PM/NM, $41 \sim 43 \%$ in LYSO, $6 \sim 8 \%$ in HDM and $1 \sim 2 \%$ in LDM.

Insulin treatment of intact hepatocytes did not induce any significant changes in the subcellular distributions of the glucose-sensitive, cytochalasin B binding activities, the immunoreactivities to the transporter specific antibodies, or the two cell surface membrane markers. These findings indicate that as much as $15 \%$ of the total hepatocyte glucose transporters occur in organelle $(\mathrm{s})$ other than the pasma membrane, most likely representing an intracellular storage pool, which is not decreased by insulin. It is concluded that the rat hepatocyte lacks the insulin-mediated, glucose transporter translocation mechanism, thus would be a valuable experimental system in which one can study the celluar and molecular basis of this deficiency.

KEY WORDS : Translocation - Subcellular distribution - Surface label · Cytochalasin B • Insulin * Hepatocyte.

\section{Introduction}

It is well known that glucose transport in hepatocytes is little affected by insulin ${ }^{1-5)}$, whereas that in adipocytes is greatly stimulated by this hormone ${ }^{6-9)}$. What makes glucose transport function sensitive to insulin in one cell type such as adipocytes, and insensitive in another such as liver cells 
is an interesting, unanswered question at this time It is worthwhile to note in this regard that constitutive(in the absence of hormonal control or basal) glucose transport activity is much(100-fold) higher in liver cells than in adipocytes, and that the adipocyte transport activity approaches the liver cell activity when insulin is present. Since insulin can only upregulate(stimulate) glucose transport activity, it is clear that insulin can regulate glucose transport only when a cell constitutively maintains sufficiently low transport activity. This emphasizes the importance of the constitutively regulated, low glucose transport activity as an integral part of the insulin-mediated glucose transport regulation mechanisms in these cells.

Both constitutive and insulin-mediated regulation of glucose transport function in mammalian cells appear to depend upon the relative distribution of glucose transporters between the plasma membrane and an intracellular storage pool. In adipocytes, it is well established that glucose transporters occur not only in the plasma membrane, but also in an intracellular storage compartment that is included in a low density microsomal fraction $^{7-9)}$. In the absence of insulin, most ( 70 to 90 $\%$ ) of the adipocyte transporter is in the storage pool with very few in the plasma membrane. Insulin stimulates glucose transport in this cell largely by redistributing(recruiting) transporter from the storage compartment to the plasma membrane 8.9). In hepatocytes, the subcellular distribution of glucose transporter is less well documented: Glucose transporter is shown to be highly concentrated in the plasma membrane ${ }^{3-5)}$. A negligible amount of glucose transporter was found in low density microsomes of hepatocytes that was detectable at the limits of resolution, and this distribution was not significantly affected by insulin ${ }^{5)}$. It is thus possible that the apparent insensitivity of the hepatocyte system could be simply due to lack of the constitutively maintained, intracellular storage pool of glucose transporter, rather than lack of insulin-mediated transporter translocation mechanism in this cell. It is also possible, on the other hand, that the hepatocyte has an intracellular glucose transporter storage pool, but lacks the insulin-mediated recruitment mechanism : Insulin-mediated glucose transporter recruitment may require a specific glucose transporter isoform(s) (insulin-regulatable glucose transporter) ${ }^{10}$, and this isoform may not occur in hepatocytes. Recent cDNA sequence analyses have indeed disclosed that two separate glucose transporters are operative in liver cells and adipocytes, and these isoforms show significant amino acid sequence differences $^{11-14)}$. Alternatively, a cell-specific factor, rather than a specific transporter isoform itself, may be essential for the insulin mediated transport recruitment, and hepatocytes may lack this factor.

In the present investigation, I examined if indeed any intracellular glucose transporter pool exists in hepatocytes, and whether this pool, if any, is affected by insulin. In adipocytes, the intracellular pool has been well studied and is known to be in a low density microsomal fraction 6.7). Although the presence of a similar intracellular glucose transporter pool in hepatocytes has been suggested ${ }^{5)}$, it has not been systematically studied. The aim of the present study was to locate and quantitate this suggested intracellular storage pool of glucose transporter in hepatocytes in the presence and absence of insulin. I measured glucose transporter in the entire hepatocyte membrane fraction based on glucose-sensitive cytochalasin B binding as well as Western blot analysis using hepatocyte glucose transporter-specific antisera binding ${ }^{14)}$. I also measured the distribution of two surface membrane markers. Our results demonstrate that up to $15 \%$ of the hepatocyte glucose transporter is in organelles other than the plasma membrane, and may be in an intracellular storage pool, yet this pool is not reduced in the presence of insulin. I conclude that the hepatocyte glucose transport system inherently lacks the in- 
sulin-mediated translocation mechanism. Hence, hepatocytes can be used to determine whether it is the lack of a specific transporter isoform, or a cell-specific factor that makes these cells deficient in insulin-induced translocation of glucose transporter.

\section{Experimental Procedures}

Materials: Cytochalasin B and Triton X-100 were purchased from Sigma. Collagenase was purchased from Worthington. Bovine albumin was from Armour Pharmaceuticals. $\left[{ }^{3} \mathrm{H}\right]$ cytochalasin B, $\mathrm{H}_{3}{ }^{32} \mathrm{PO}_{4}$ and $\mathrm{NaB}^{3} \mathrm{H}_{4}$ were obtained from New England Nuclear. ${ }^{125}$ I-labeled protein $\mathrm{A}$ was from Amersham. Insulin(porcine crystalline) was a gift from Eli Lilly \& Co. Percoll was obtained from Pharmacia Fine Chemicals. All other chemicals were standard commercial products of reagent grade quality.

Isolation of heptocytes : Rats (Sprague-Dawley, 150 200g body weight), were anesthetized with sodium pentabarbital ( $50 \mathrm{mg} / \mathrm{kg}$ body weight) and subjected to liver perfusion ${ }^{15)}$. The liver was first perfused with $400 \mathrm{ml} \mathrm{Ca}^{++}$-free Hank's buffer, $\mathrm{pH}$ 7.4, containing EGTA( $5 \mathrm{mM})$ and D-glucose ( 5.5 $\mathrm{mM}$ ), followed by perfusion with $200 \mathrm{ml}$ of Hank's buffer containing $\mathrm{CaCl}_{2}(2 \mathrm{mM})$ and collagenase $(100 \mathrm{mg} / 150 \mathrm{ml})$. The liver was removed, and transferred(holding it at the hepatic vein trunk) into a petri dish containing $50 \mathrm{ml}$ of the collagenase buffer. After incubation at $37^{\circ} \mathrm{C}$ for $10 \mathrm{~min}$, cells were filtered through a $60 \mu \mathrm{m}$ nylon mesh and washed 4 times in Hank's buffer $(40 \mathrm{ml})$ at $4^{\circ} \mathrm{C}$. The resulting isolated cells were resuspended in Krebs-Ringer phosphate buffer containing $2 \mathrm{mM}$ pyruvate and $1 \%$ BSA, with readjustment at $\mathrm{pH}$ 7.4. After $10 \mathrm{~min}$ incubation at $37^{\circ} \mathrm{C}$, cells were separated by centrifugation and resuspended into the same suspension buffer at a concentration of $1 \sim 2 \times 10^{6} \mathrm{cells} / \mathrm{ml}$. Hepatocytes were counted by a Coulter electronic particle counter. One rat liver typically gave $10 \sim 12 \times 10^{6}$ isolated hepatocytes or $3 \sim 4 \mathrm{ml}$ of packed cells.

Subcellular fractionation: The procedure was essentially that of Fleischer \& Kervina ${ }^{16)}$. The suspension of hepatocytes was spun at $10000 \times \mathrm{g}$ for $10 \mathrm{~min}$ at $4^{\circ} \mathrm{C}$ using a Sorvall centrifuge. One $\mathrm{ml}$ of packed cells were resuspended in $5 \mathrm{ml}$ of homogenizing solution(Sucrose, 0.25M; HEPES, 10 $\mathrm{mM}, \mathrm{pH} 7.5$ ) and homogenized at $2000 \times \mathrm{rpm}$ ( $3 \sim$ 4 strokes). The homogenate was centrifuged at $200,000 \times \mathrm{g}$ for $180 \mathrm{~min}$ and the pellet(PO) was used as total particulate fraction. Individual subcellular membrane fractions were separated as follows. The homogenate was centrifuged at 200 $x_{\mathrm{g}}$ for $4 \mathrm{~min}$. The supernatant was recovered using a pasteur pipet and saved for further subfractionation. The pellet was resuspended in $3 \mathrm{ml}$ of homogenizing solution and homogenized as above. The homogenate was centrifuged at $200 \times \mathrm{g}$ for $4 \mathrm{~min}$ and the supernatant was pooled with the previous supernatant. Homogenization and centrifugation were repeated $4 \sim 5$ times using $2 \mathrm{ml}$ of homogenizing solution until all cells are disrupted under a light microscope. The whole supernatant was centrifuged at $1450 \times \mathrm{g}$ for $10 \mathrm{~min}$. The resultant pellet( $\mathrm{P} 1)$ is known to contain plasma membrane, nucleus and mitochondria(PM/NM) 16). The supernatant was centrifuged at $25000 \times \mathrm{g}$ for $10 \mathrm{~min}$. The resulting pellet $(\mathrm{P} 2)$ is known to contain abundant lysosomes(lysosome enriched fraction or LYSO) ${ }^{16)}$. The supernatant was centrifuged at $48000 \times \mathrm{g}$ for $30 \mathrm{~min}$ yielding a pellet(P3) of high density microsomal membranes(HDM). The supernatant was then centrifuged at 200,000 $\times \mathrm{g}$ for $180 \mathrm{~min}$, yielding a pellet $(\mathbf{P} 4)$ of low density microsomal membranes(LDM). Thus, our subcellular fractionation schemes are similar to that of Ciaraldi et al. ${ }^{5)}$, except that we did not discard any particulate fractions.

The plasma membranes were purified from the $1450 \times \mathrm{g}$ pellet $(\mathrm{P} 1)$ by employing the percoll density gradient centrifugation method of Belsham 
et al. ${ }^{17)}$ and sucrose density gradient centrifugation method of Lesko et al ${ }^{18)}$. P1 was resuspended in $3 \mathrm{ml}$ of homogenizing solution and applied on 2 tubes of percoll density gradient solution(percoll $6.3 \mathrm{ml}$; homogenizing buffer $28.8 \mathrm{ml}$; percoll solution $0.9 \mathrm{ml}$ ), and then centrifuged at 10 , $000 \times \mathrm{g}$ for $15 \mathrm{~min}$, which gave 2 layers. Percoll solution contained $2 \mathrm{M}$ sucrose, $8 \mathrm{mM}$ EGTA and $80 \mathrm{mM}$ Tris-Cl. The upper layer containing plasma membranes(PM) was collected and washed with homogenizing solution to remove percoll by centrifugation at $80,000 \times \mathrm{g}$ for $30 \mathrm{~min}$. Centrifugation was repeated 5 times until percoll was removed completely. The pellet was resuspended in homogenizing solution and stored at $-20^{\circ} \mathrm{C}$ until use. The lower pellet containing nucleus and mitochondria (NM) was washed 2 times with homogenizing solution and stored at $-20^{\circ} \mathrm{C}$ until use. For the sucrose density gradient centrifugation method, $\mathrm{P} 1$ was resuspended in $3 \mathrm{ml}$ of homogenizing solution. The suspension $(2.6 \mathrm{ml})$ was mixed with $5.67 \mathrm{ml}$ of $70 \%$ ice cold sucrose solution. The mixture was divided equally into two SW-27 ultra clear tubes. Over each suspension was layered $10 \mathrm{ml}$ of $45 \%$ sucrose, then $12.5 \mathrm{ml}$ of $41 \%$ sucrose and finally $5 \mathrm{ml}$ of $37 \%$ sucrose. After centrifugation at $65,000 \times \mathrm{g}$ for 2 hours the upper layer was collected and diluted 6 times with homogenizing buffer and spun again $200,000 \times \mathrm{g}$ for 2 hours. The resultant pellet was used as partially purified plasma membranes(PM). The lower pellet was washed with homogenizing solution and saved for nucleus and mitochondrial membranes(NM).

Cytochalasin B binding assay: Equilibrium binding of cytochalasin $\mathrm{B}$ to particulate fractions was determined by a centrifugation method ${ }^{19)}$. Vesicles were incubated with $\left[{ }^{3} \mathrm{H}\right]$ cytochalasin B at specified concentrations, with or without additives, for $30 \mathrm{~min}$ at room temperature and centrifuged for $30 \mathrm{~min}$ at $4^{\circ} \mathrm{C}$ at $260,000 \times \mathrm{g}$. Pellets and supernatants were recovered free of cross-contamination. Radioactivities of both pellets and su- pernatants were assayed, from which bound ligands were calculated in percent of the total ligands in the assay mixture. Typically, binding assay mixture contained $2 \sim 4 \mathrm{mg}$ protein equivalent particulates in a buffer containing $10 \mathrm{mM}$ Tris $\mathrm{HCl}(\mathrm{pH}$ 7.4), $1.0 \%$ ethanol and specified amounts of ligands in a final volume of $1.0 \mathrm{ml}$. In displacement experiments, the assay mixture also included varying amounts of specified additives. Binding data were analyzed by Scatchard plots ${ }^{20)}$.

Western blot analysis: Aliquots of hepatocyte subcellular fractions $(20 \sim 50 \mu \mathrm{g}$ protein $)$ were electrophoresed on $10 \%$ acrylamide gel in NaDo$\mathrm{dSO}_{4}$, then electroblotted onto nitrocellulose membrane. The blots were probed with antibodies raised against the carboxyl-terminal end(a.a. 509 522) of the rat hepatocyte glucose transporter ${ }^{14)}$ and detected with ${ }^{125} \mathrm{I}$ labeled protein $\mathrm{A}(1$ $\mu \mathrm{Ci})$.

Plasma membrane marker assays: 5'-nucleotidase(EC 3.1,3.5) activities were assayed by the method of Avruch and Wallach ${ }^{21)}$ in the presence of $0.05 \%$ Triton X-100 and 5mM 2'-3'-AMP to inhibit nonspecific phosphatases.

Cell surface labeling with sodium borohydride : $1.0 \sim 1.4 \mathrm{ml}$ of packed hepatocytes were washed three times with $0.9 \% \mathrm{NaCl}$ in $10 \mathrm{mM}$ sodium acetate $(\mathrm{pH} 5.5)$ and resuspended in $5 \mathrm{ml}$ of the washing buffer. $20 \mu$ of sodium meta-periodate stock solution $10.7 \mathrm{mg} \mathrm{NaIO}_{4}$ dissolved in 100 $\mu 1$ ) was added to this suspension (a final concentration of $2 \mathrm{mM}$ ), and incubated for $30 \mathrm{~min}$ on ice. Cells were then washed 3times with $40 \mathrm{ml}$ Krebs-Ringer phosphate buffer(pH 7.4) and resuspended in $2 \mathrm{ml}$ of the same buffer. $100 \mu \mathrm{l}$ of 100 $\mathrm{mM} \mathrm{NaBH}{ }_{4}$ solution containing a trace amount of $\mathrm{NaB}^{3} \mathrm{H}_{4}(1 \mu \mathrm{Ci})$ was added to this suspension, and the mixture was kept for $60 \mathrm{~min}$ on ice. Cells were finally washed twice with Krebs-Ringer phosphate buffer( $\mathrm{pH}$ 7.4) prior to homogenization and subfractionation. 


\section{Results}

Cytochalasin B binding to hepatocyte total particulates: Equilibrium binding of cytochalasin B to the total hepatocyte particulates revealed several saturable binding components (Fig. 1). A large portion (more than $85 \%$ ) of the saturable binding was inhibited by an excess $\left(10^{-4} \mathrm{M}\right)$ of cytochalasin E, a well known cytoskeletal marker ${ }^{23)}$. Only a small, but significant portion of the saturable binding was displaced by an excess $(500 \mathrm{mM})$ of Dglucose(Fig. 1). L-glucose did not displace this component(not illustrated), indicating that the

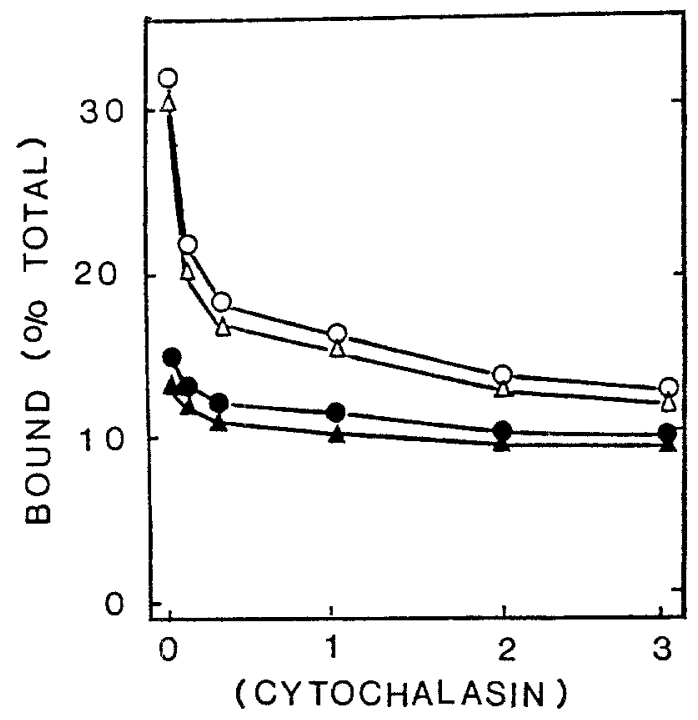

Fig. 1. Equilibrium binding of cytochalsin $B$ to total particulates of rat hepatocytes as a function of cytochalasin B concentration, in the absense $(\mathrm{O})$, and in the presence of $500 \mathrm{mM}$ D-glucose $(\triangle), 10^{-4} \mathrm{M}$ cytochalasin $\mathrm{E}(\mathrm{O})$ or $500 \mathrm{mM}$ D-glucose plus $10^{-4} \mathrm{M}$ cytochalasin $\mathrm{E}(\boldsymbol{\Delta})$. Aliquots of total particulates $(2.8 \mathrm{mg}$ protein) were incubated for $20 \mathrm{~min}$ at $21^{\circ} \mathrm{C}$ in a total of $1.0 \mathrm{ml}$ buffer containing $0.1 \mu \mathrm{Ci}$ of $\left[{ }^{3} \mathrm{H}\right]$ cytochalasin $\mathrm{B}$ together with cytochalasin $\mathrm{B}$ and other ligands at specified concentrations, as detailed in the Experimental Procedures. The radioactivities associated with particulates were expressed in \% of the total radioactivities in the binding assay mixture, and are plotted as a function of cytochalsin $\mathrm{B}$ concentration in the mixture.

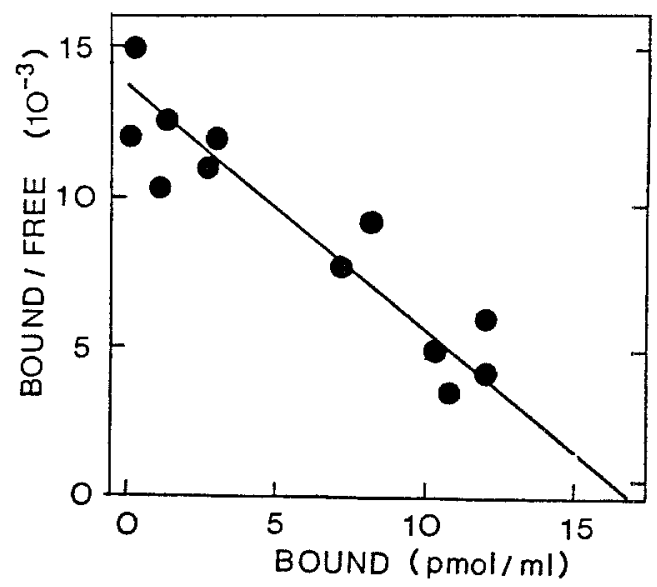

Fig. 2. Derived Scatchard plots of D-glucose-sensitive, cytochalasin B binding components of total particulates of rat hepatocytes measured in the presence of $10^{-4} \mathrm{M}$ cytochalsin $\mathrm{E}$. The glucose-sensitive binding components were calculated by the differences in the ligand binding observed in the absence and in the presence of $500 \mathrm{mM}$ D-glucose for each ligand concentration. The straight line shown is the best fit to the data and represents $K_{d}$ of $1.3 \times 10^{-6} \mathrm{M}$ and $B_{t}$ of 6.1 $\mathrm{pmol} / \mathrm{mg}$ protein.

displacement was specific to D-glucose. This glucose-sensitive binding component was also observable in the presence of an excess cytochalasin E(Fig. 1). In derived Scatchard plots (Fig. 2), this glucose-sensitive binding behaved as a single binding component with a $\mathrm{K}_{\mathfrak{d}}$ of $1.68 \pm 0.63 \times 10^{-6} \mathrm{M}$ and $\mathrm{B}_{\mathrm{t}}$ of $6.8 \pm 1.2 \mathrm{pmol} / \mathrm{mg}$ protein(mena \pm S.D., $n=5)$. Values for the $K_{d}$ and $B_{t}$ essentially identical to these were also observed in the absence of cytochalasin E(not illustrated), indicating there is no significant interaction between the glucose-sensitive binding and the cytochalasin E-sensitive binding sites.

The glucose-sensitive cytochalsin $\mathrm{B}$ binding has been described in many animal cells ${ }^{8.19 .23 .24)}$ including hepatocytes ${ }^{3.5)}$ in relation to glucose transporter. The glucose-sensitive cytochalasin B binding in hepatocytes, however, was shown to be unique in that the binding affinity is almost tenfold lower compared with that in other cells such 
as human erythrocytes ${ }^{19)}$, lymphocytes ${ }^{24)}$, and adipocytes $^{8)}$. The present data confirm these findings. This lower binding affinity is consistent with the reduced affinity of this ligand as an inhibitor of glucose transport in hepatocytes ${ }^{3.5)}$.

Subcellular distributon of D-glucose-sensitive cytochalasin B binding sites: By differential centrifugation, I separated hepatocyte homogenates into four particulate fractions, P1, P2, P3 and P4. These fractions were similar to the plasma membrane/nuclei/mitochondria-enriched fraction(PM/ NM), lysosome-enriched fraction(LYSO), high density microsomes(HDM) and low density mic-

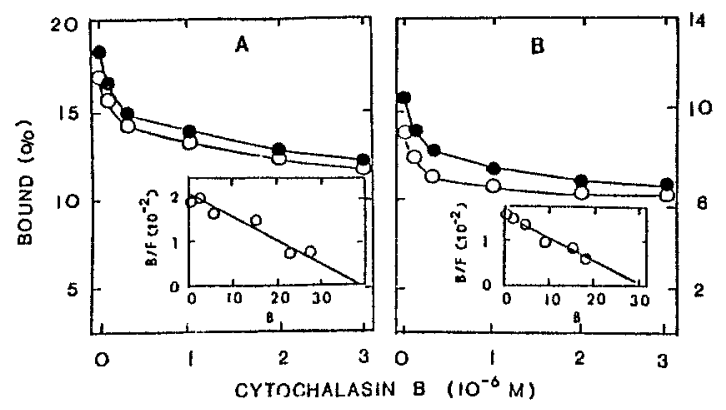

Fig. 3. Equilibrium binding of cytochalsin $B$ to the high (A) and low (B) density microsomes of rat hepatocytes as a function of cytochalsin B concentration in the absence ( $)$ and in the presence (O) of $500 \mathrm{mM}$ D-glucose, both in the presence of $10^{-5} \mathrm{M}$ cytochalasin $\mathrm{E}$.

Experimental protocol and data presentation are identical to those of Figures 1 and 2. The amounts of protein used per tube were $3.7 \mathrm{mg}$ for HDM and $2.9 \mathrm{mg}$ for LDM. Inserts are Scatchard plots of glucose-sensitive binding components.
rosome(LDM), respectively, of Fleischer and Kervina ${ }^{16)}$. Particular care was paid to recover all the particulates possible, rather than to purify any particular compartments. When so specified, P1 (PM/NM enriched fraction) was further fractionated to separate PM and NM fractions, which resulted in a significant $(50 \%$ or more) loss in recovery. In each of these fractions, equilibrium binding of cytochalasin $\mathrm{B}$ was measured in the presence of $10^{-4} \mathrm{M}$ cytochalasin $\mathrm{E}$, with and without $500 \mathrm{mM}$ D-glucose. The glucose-sensitive cytochalasin B binding sites were assessed based on derived Scatchard analyses. Figure 3 illustrates typical results of such experiments. The overall results are summarized in Table 1. When expressed in terms of $B_{t}$ (the total binding sites/mg protein), the glucose-sensitive binding sites are found not only in PM/NM, but also in HDM, and LDM. In PM/NM, they are mostly in PM, but very little in NM. When the same data were expressed in terms of total contents (rather than concentrations) in each particulate fraction, only $47 \%$ of the total cellular content was in PM/NM, and less than $10 \%$ was found in LDM. The remaining $45 \%$ was recovered in LYSO and HDM. It is clear that a large portion ( $50 \%$ or more) of the glucosesensitive cytochalsin B binding sites of hepatocytes resides in subcellular fractions other than $\mathrm{PM} / \mathrm{NM}$.

Subcellular distributions of two plasma membrane markers: In order to estimate how much

Table 1. Relative distribution of glucose-sensitive cytochalasin B binding activities in hepatocyte subcellular particulate fractions

\begin{tabular}{llccccc}
\hline & & Protein & \multicolumn{2}{c}{$\begin{array}{c}\text { Glucose-sensitive cytochalasin B } \\
\text { binding parameters }\end{array}$} & $\begin{array}{c}\text { No.of } \\
\mathrm{K}_{\mathrm{d}}\end{array}$ & $\begin{array}{c}\text { Determin- } \\
\text { ations }\end{array}$ \\
\hline Fractions & (\% total particulates) & (pmol/mg protein) & $(\%)$ & $\left(10^{-6} \mathrm{M}\right)$ & 8 \\
\hline P1 & PM/NM & $48.8 \pm 8.2$ & $6.7 \pm 0.91$ & 46.9 & $1.78 \pm 0.81$ & 8 \\
& PM & $6.2 \pm 1.9$ & $36.4 \pm 1.38$ & - & $1.75 \pm 0.96$ & 4 \\
& NM & $17.6 \pm 2.1$ & $0.8 \pm 0.12$ & - & $1.4 \pm 1.3$ & 4 \\
P2 & LYSO & $30.7 \pm 3.6$ & $6.8 \pm 1.54$ & 28.8 & $1.12 \pm 0.73$ & 8 \\
P3 & HDM & $12.6 \pm 1.5$ & $9.1 \pm 1.63$ & 16.2 & $1.48 \pm 0.61$ & 8 \\
P4 LDM & $7.4 \pm 1.1$ & $7.6 \pm 1.2$ & 7.9 & $1.93 \pm 0.63$ & 8 \\
\hline
\end{tabular}


Tabłe 2. Relative distribution of $\mathrm{NaBH}_{4}$ surface label and 5'-nucleotidase activity in rat hepatocyte particulate fractions

\begin{tabular}{|c|c|c|c|c|c|c|}
\hline \multicolumn{2}{|c|}{$\begin{array}{l}\text { Particulate } \\
\text { Fractions }\end{array}$} & \multirow{2}{*}{$\begin{array}{l}\text { Protein } \\
(\% \text { total }) \\
46.9 \pm 6.1\end{array}$} & \multirow{2}{*}{$\begin{array}{c}{\left[\begin{array}{c}{\left[{ }^{3} \mathrm{H}\right] \mathrm{NaBH}_{4}} \\
\text { (cpm in } 10^{3} \mathrm{mg} \text { protein) }\end{array}\right.} \\
3.3 \pm 1.1\end{array}$} & \multirow{2}{*}{$\frac{(\%)}{48.7}$} & \multirow{2}{*}{$\begin{array}{c}\begin{array}{c}\text { '-nucleotidase } \\
(\mu \mathrm{mol} / \mathrm{h} / \mathrm{mg} \text { protein })\end{array} \\
0.97 \pm 0.12\end{array}$} & \multirow{2}{*}{$\frac{(\%)}{49.8}$} \\
\hline P1 & $\mathrm{PM} / \mathrm{NM}$ & & & & & \\
\hline & PM & $6.6 \pm 1.5$ & $16.8 \pm 1.3$ & - & $5.94 \pm 0.63$ & - \\
\hline & NM & $16.8 \pm 1.3$ & $2.8 \pm 1.5$ & - & $0.06 \pm 0.01$ & - \\
\hline P2 & LYSO & $32.5 \pm 2.8$ & $3.9 \pm 0.8$ & 41.0 & $1.23 \pm 0.12$ & 43.0 \\
\hline P3 & HDM & $14.9 \pm 1.6$ & $1.8 \pm 1.3$ & 8.3 & $0.36 \pm 0.04$ & 6.1 \\
\hline P4 & LDM & $5.8 \pm 0.8$ & $1.0 \pm 1.2$ & 1.8 & $0.17 \pm 0.02$ & 1.1 \\
\hline
\end{tabular}

of the cytochalasin B binding found in each of these fractions is due to contamination by the plasma membrane, we have measured the distribution of two well known plasma membrane markers, sodium borohydride surface label and 5'nucleotidase activity, in these fractions(Table 2). When intact hepatocytes were labeled with $\mathrm{Na}-$ $\left[{ }^{3} \mathrm{H}\right]$-borohydride prior to fractionation, the surface label was concentrated not only in PM/NM but also in LYSO, indicating that LYSO contains a large portion of the plasma membrane. The cell surface label, on the other hand, was significantly less concentrated in HDM, indicating that the plasma membrane contamination in HDM is much less than in LYSO. Very little surface label was found in LDM, on the other hand, indicating no significant contamination by the plasma membrane in LDM. Essentially, a similar subcellular distribution pattern was observed for 5'-nucleotidase(Table 2).

Estimation of a non-plasma membrane glucose transporter pool : Comparison of the \% distribution of the glucose-sensitive cytochalasin B bin- ding sites with the \% distribution of the two plasma membrane markers for each subcellular fraction(Table 3) reveals that practically all of the glucose transporter found in PM/NM and LYSO is accountable as of plasma membrane origin. In HDM, however, at maximum only about half ( $6 \sim 8$ versus $16 \%$ ) of its glucose transporter content could be accounted for as of the plasma membrane origin. In LDM, only a very small portion $(1 \sim 2$ versus $8 \%)$ of the glucose transporter content could be accounted for by the plasma membrane contamination. These considerations indicate that as much as 14 to $15 \%$ of the glucose transporters in hepatocytes reside in a subcellular compartment other than the plasma membrane.

Lack of insulin effect on the subcellular distribution of glucose transporter in hepatocytes: Isolated hepatocytes were first incubated in the absence and in the presence of insulin $(7 \mathrm{nM})$ for 30 min at $37^{\circ} \mathrm{C}$, then subjected to the standard subcellular fractionation, after which glucose-sensitive cytochalasin $\mathrm{B}$ binding activities were determined for each fraction(Table 3). It is clear that insulin

Table 3. Effects of insulin treatment on subcellular distribution of glucose-sensitive cytochalsin B binding activities. $B_{t}$ are in $\mathrm{pmol} / \mathrm{mg}$ protein. Also showed are $\mathrm{K}_{\mathrm{d}}$ in $10^{-6} \mathrm{M}$. Both parameters were assessed based on Scatchard analysis for means \pm SEM with the number of determinations

\begin{tabular}{|c|c|c|c|c|c|}
\hline \multirow{3}{*}{$\begin{array}{l}\text { Particulate } \\
\text { fractions }\end{array}$} & \multirow{2}{*}{\multicolumn{5}{|c|}{$\begin{array}{l}\text { Glucose-sensitive cytochalasin B binding } \\
\text { with insulin } \\
\text { without insulin }\end{array}$}} \\
\hline & & & & & \\
\hline & $B_{t}$ & $\mathrm{~K}_{\mathrm{d}}$ & $\mathrm{B}_{\mathrm{t}}$ & $\mathrm{K}_{\mathrm{d}}$ & $\mathrm{n}$ \\
\hline $\mathrm{P} 1 \quad \mathrm{PM} / \mathrm{NM}$ & $6.8 \pm 1.2$ & $(1.52 \pm 1.1)$ & $7.1 \pm 1.3$ & $(1.21 \pm 0.73)$ & 3 \\
\hline P2 LYSO & $7.1 \pm 1.3$ & $(1.61 \pm 1.2)$ & $6.8 \pm 1.6$ & $(1.33 \pm 0.83)$ & 3 \\
\hline P3 HDM & $8.9 \pm 1.7$ & $(1.43 \pm 1.2)$ & $9.2 \pm 1.7$ & $(1.51 \pm 0.81)$ & 5 \\
\hline P4 LDM & $7.5 \pm 1.4$ & $(1.83 \pm 0.81)$ & $7.4 \pm 1.0$ & $(1.68 \pm 0.61)$ & 5 \\
\hline
\end{tabular}


did not cause any significant change in the glucose-sensitive cytochalsin $\mathrm{B}$ binding activities of any subcellular membrane fractions including HDM and LDM. In parallel experiments, insulin effects on 5'-nucleotidase activities of HDM and LDM fractions were assessed. The results of these assessments (data not illustrated) showed that insulin has no effect on the activities of this plasma membrane marker in either of these microsomal fractions.

Possible insulin effects on the subcellular distribution of glucose transporter in hepatocytes

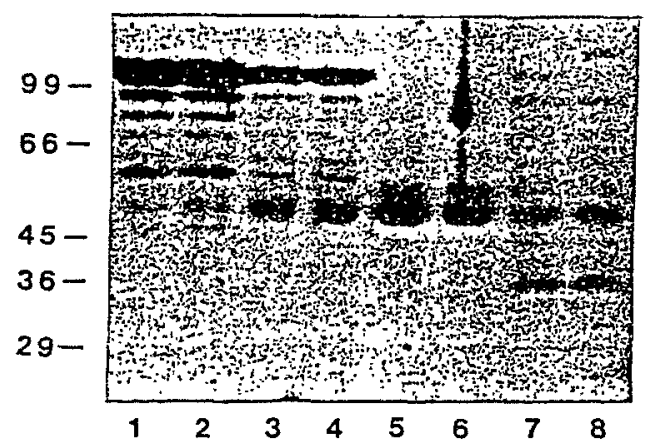

Fig. 4. Western blot analysis of hepatocyte glucose transporter in different subcellular membrane fractions: Aliquots of subcellular particulate fractions (30 $\mu \mathrm{g}$ protein each) were subjected to $\mathrm{NaDodSO}_{4}$ polyacrylamide gel electrophoresis, immunoblotting and autoradiographic detection. PM/NM (lanes 1 and 2), LYSO (lanes 3 and 4), HDM (lanes 5 and 6), LDM (lanes 7 and 8). Without insulin treatment (lanes $1,3,5$ and 7) and with insulin treatment (lanes $2,4,6$ and 8). Positions of molecular weight markers are shown on the left margin in $\mathrm{kDa}$.

were also studied by Western blot analysis using antisera specific to the hepatocyte glucose transporter $^{14)}$ (Fig. 4). Several points should be noted here. First, the basal(in the absence of insulin) subcellular distribution of the immunoreactivity was very similar to that of glucose-sensitive cytochalasin B equilibrium binding data, showing considerably more binding in PM/NM, LYSO and HDM than in LDM. Exact quantitative correlation was not possible because relative blotting ef- ficiencies of the antibodies to different subcellular fractions are not known. Second, glucose transporters in the PM/NM and LYSO fractions appear much more aggregated than in the HDM and LDM fraction. The significance of this observation is not immediately clear. Significant amounts of immunoreactivity were found in a number of bands which cannot be assigned as either a monomer or a dimer of glucose transporter, and may in part represent nonspecific crossreactivities of the antisera used. Lastly, and most importantly, it is quite clear that none of these reactivities was affected by insulin treatment, including those of HDM and LDM. It is particularly significant to note the absence of insulin effect in LDM, where the plasma membrane contamination is minimal, and most likely intracellular glucose transporters reside.

\section{Discussion}

One major aim of the present study was to determine if indeed there is an intracellular storage pool of glucose transporter in hepatocytes similar to those found in insulin-responsive systems such as isolated rat adipocytes and 3T3-L1 adipocytes ${ }^{6}$. 26). The presence of such a pool in hepatocytes was first suggested by Ciaraldi et al. ${ }^{5)}$. They found a small amount of glucose-sensitive cytochalasin $B$ binding activity ( $4 \mathrm{pmol} / \mathrm{mg}$ protein) in a low density microsomal fraction of rat hepatocytes. However, they cautioned that the amount observed in this fraction was at the limit of resolution of their assay method. They also noted that the plasma membrane contamination in this fraction could be as high as $16 \%$, and the transporter concentration in the plasma membrane was at least ten-fold higher ( $42 \mathrm{pmol} / \mathrm{mg}$ protein) than in the low density microsomes, so the amount of glucose transporter in the low density microsome fraction could be totally accounted for by the plasma membrane contamination. Furthermore, they could 
not detect any glucose-sensitive cytochalasin B binding in the high density microsomes. In the same fraction, however, a significant amount of glucose-sensitive cytochalasin $\mathrm{B}$ binding activity was observed in the present study. The reason for this discrepancy is not immediately clear. They did not examine the lysosome-enriched fraction, which appeared in the present study to be a major source of glucose transporter in hepatocytes.

Based on the glucose-sensitive cytochalasin B equilibrium binding assay, I demonstrated here that a large amount of glucose transporter occurs in subcellular fractions other than the PM/NM fraction, namely, LYSO, HDM and LDM(Table 1). The finding of the transporter in LYSO and HDM is in fact not surprising, since a significant portion of the plasma membrane is known to be included in LYSO and HDM in the subcellular fractionation scheme adopted here ${ }^{16)}$. The data on the subcellular distribution of the two plasma membrane markers (Table 2) also demonstrate a large contamination by the plasma membrane in these two fractions. Significant amounts of glucose transporter activity was found even in purified lysosomal membranes(tritosomes) ${ }^{27}$, indicating that not all of the glucose transporter found in LYSO fraction may come from the plasma membrane contamination. The finding of the transporter in LDM, on the other hand, was not expected, since plasma membrane contamination in this fraction is known to be negligible in the fractionation scheme ${ }^{16)}$ Which is supported by the plasma membrane marker data(Table 2) in the present study. If one considers the total content. rather than concentration, of glucose transporter in each fraction, almost $50 \%$ of the total hepatocyte glucose transporter is in the PM/NM fraction. and $8 \%$ of the glucose transporter is in the LDM fraction(Table 1). A similar relative distribution of glucose transporter among the different subcellular fractions was found with the Western blot analysis using hepatocyte glucose transporter-spe- cific antibodies(Fig. 4)

No specific biochemical or cytological identification procedure for the intracellular glucose transporter pool is currently available. Consequently, it is difficult. if not impossible, to establish the presence of the intracellular pool of glucose transporter when it is small. We define the glucose transporter content in each fraction that cannot be accounted for by possible plasma membrane contamination as the intracellular storage pool. A comparison of the \% subcellular distribution of the glucose-sensitive cytochalasin B binding site(Table 1, column 4) with the corresponding \% subcellular distributions of the two plasma membrane markers(Table 2, columns 4 and 6) shows that $49 \sim 62 \%$ and $77 \sim 86 \%$ of the glucose transporter content in HDM and LDM, respectively, are not accounted for by possible plasma membrane contamination, and by definition, represent intracellular glucose transporter pool. This conclusion is consistent with the observation that glucose transporter is in fact more concentrated in HDM and LDM than in the other fraction. with the exception of the partially purified PM (Table 1). The hepatocyte HDM has been shown to contain a significant amount of Golgi membrane ${ }^{16)}$. Morphological studies ${ }^{26)}$ have revealed that most of the intracellular glucose transporters in 3T3-L1 adipocytes are associated with the tubulovesicular structure at the trans side of the Golgi apparatus. It is possible that during cell homogenation this structure may go to HDM in hepatocytes. Glucose transporter in LDM, on the other hand, may be in endocytic vesicles. In the fractionation protocol used here, the intracellular compartment of the hepatocyte glucose transporter fractionates into the high and low density microsomal fractions corresponding to $8 \sim 10 \%$ and $6 \sim 7 \%$ of the total hepatocyte glucose transporter, respectively. It is estimated that as much as 12 to $17 \%$ of the hepatocyte glucose transporter exist in a compartment other than the plasma memb- 
rane, and may represent an intracellular pool in this cell.

The other major aim of the present study was to examine if the glucose transporter in the intracellular pool is translocated to the plasma membrane in response to insulin in hepatocytes as in adipocytes. The data from both the cytochalasin $B$ binding assay(Table 3) and the immunoblot assay(Fig. 4) clearly demonstrate that insulin causes no detectable change in the amounts of glucose transporter in any subcellular fractions. The lack of an insulin effect on the glucose transporter contents of the HDM and LDM fractions is particularly important in this regard. It is these fractions that should be reduced significantly (by as much as 50 and $80 \%$ ) if a genuine, intracellular glucose transporter pool existed and responded to the insulin-mediated translocation mechanism. It is, therefore, concluded that insulin does not translocate the glucose transporter from the intracellular pool to the plasma membrane in hepatocytes.

As insulin can only upregulate glucose transport function, a constitutively maintained, low level of the transport function is a prerequisite to insulin-mediated regulation of glucose transport function. In the recruitment hypothesis ${ }^{8.9)}$, a constitutively maintained, intracellular storage pool of glucose transporter is an essential feature of the insulin action, and may in fact be a part of the insulin regulatory mechanism. If there is no intracellular pool, there could be no recruitment, even in the presence of all other suggested requirements for the insulin-mediated translocation such as insulin-responsive transporter isoforms or putative, insulin-sensitivity conferring cellular factors (see Introduction). It would be interesting in this regard to study a cell which has an intracellular glucose transporter pool much larger than hepatocytes, yet its glucose transport function is not stimulated by insulin.

\section{Conclusion}

The present study unequivocally demonstrated that the hepatocyte glucose transport system is inherently deficient of the insulin-mediated translocation mechanism, and that the apparent insensitivity of this system to insulin is not due to low resolution in detecting changes in the intracellular pool size of glucose transporter. Hepatocytes will provide a valuable experimental system in which to study the nature of the defect that makes the glucose transport system in this cell insulin-insensitive, which may in turn provide important insight into understanding the cellular and molecular mechanisms underlying the insulin-mediated transport recruitment.

\section{References}

1) Baly LD and Horuk R:The biology and biochemistry of the glucose transporter. Biochim Biophys Acta $1988: 947: 571-590$

2) Craik JD and Elliot KRF : Kinetics of 3-0-methyl$D$-glucose transporter in isolated rat hepatocytes. Biochem J $1979: 182: 503-508$

3) Axelrod JD and Pilch PF : Unique cytochalasin $B$ binding characteristics of the hepatic glucose carrier. Biochem J $1983: 22: 2222-2227$

4) Elliott KRF, Bate AJ and Craik JD : Specificity of the rat hepatocyte monosaccharide transporter. Int $J$ Biochem $1984: 16: 1251-1253$

5) Ciaraldi TP, Horuk $R$ and Matthaei S : Biochemical and functional characterization of the liver glucose transport system. Biochem J $1986: 240: 115$ 123

6) Simpson IA, Yver DP, Hissin PJ, Wardzala LJ, Karnieli E, Salans LB and Cushman SW: Insulinstimulated translocation of glucose transporters in the isolated rat adipose cell : Characterization of subcellular fractions. Biochim Biophys Acta 1983: $763: 393-407$

7) Zorzano A, Wilkinson W, Kotliar N, Thoidis G, Wadzinski BE, Ruoho AE and Pilch PF : Insulin- 
regulated glucose uptake in rat adipocytes is mediated by two transporter isoforms present in at least two vesicle populations. J Biol Chem 1989:264: 12358-12363

8) Cushman SW and Wardzala LJ : Potential mechanism of insulin action on glucose transport in the isolated rat adipose cell : Apparent translocation of intracellular transport systems to the plasma membrane. J Biol Chem $1980: 255: 4758-4762$

9) Suzuki $\mathrm{K}$ and Kono $\mathrm{T}$ : Evidence that insulin causes translocation of glucose transport activity to the plasma membrane from an intracellular storage site. Proc Natl Acad Sci USA 1980:77:2542-2545

10) James DE, Bronw R, Navarro J and Pilch PF : Insulin-regulatable tissues express a unique insulinsensitive glucose transport protein. Nature 1988 : $333: 183-185$

11) James $D E$, Strube $M$ and Mueckler $M$ : Molecular cloning and characterization of an insulin-regulatable glucose transporter. Nature $1989: 333: 83-87$

12) Birnbaum MJ : Identification of a novel gene encoding an insulin-responsive glucose transporter protein. Cell 1989:57:305-315

13) Fukumoto $H$, Seino $S$, Imura $S$, Seino $Y$, Eddy RL, Fuklushima Y, Byers MG, Shows YB and Bell GI: Sequence, tissue distribution and chromosomal localization of m-RNA encoding a human glucose transporter-like protein. Proc Natl Acad Sci USA $1988: 85: 5434-5438$

14) Thorens B, Sarkar HK, Kaback HR and Lodish $\mathrm{HF}$ : Cloning and functional expression in bacteria of a novel glucose transporter present in liver, intestine, kidney and $\beta$-pancreatic islet cells. Cell 1988 : $55: 281-290$

15) Seglen PO : Preparation of rat liver cells : III. Enzymatic requirements for tissue dispersion. Exp Cell Res 1973:82：391-398

16) Fleischer $S$ and Kervina $M:$ Subcellular fractionation of rat liver. Methods Enzymol 1974:31:6-41

17) Belsham GJ, Denton RM and Tanner MJA : Use of a novel rapid preparation of fat-cell plasma membranes employing percall to investigate the effects of insulin and adrenaline on membrane protein phosphorylation within intact fat-cells. Biochem $J$ $1980: 192: 457-467$

18) Lesko L, Donlon M, Marinetti GV, Hare JD : A rapid method for the isolation of rat liver plasma membranes using an aqueous two-phase polymer system. Biochim Biophys Acta $1973: 311: 173-178$

19) Jung $C Y$ and Rampal $A L$ : Cytochalasin $B$ binding sites and glucose transport carrier in human erythrocyte ghosts. J Biol Chem 1977:252:5456-54 63

20) Scatchard G: The attractions of proteins for small molecules and ions. Ann NY Acad Sci 1949:51: 660-666

21) Avruch JA and Wallach DFH : Preparation and properties of plasma membrane and endoplasmic reticulum fragments from isolated rat fat cells. Biochim Biophys Acta $1971: 233: 334-347$

22) Lowry $\mathrm{OH}$, Rosenbrough NJ, Farr AL and Randall $\mathrm{RJ}:$ Protein measurement with the folin phenol reagent. J Biol Chem 1951:193:265-275

23) Lin S, Santi DV and Spudich JA : Biochemical studies on the mode of action of cytochalasin B. J Biol Chem 1974: $249: 2268-2274$

24) Mookerjee BK, Cuppoletti J, Rampal AL and Jung CY: The effects of cytochalasins on lymphocytes: Identification of distinct cytochalasin-binding sites in relation to mitogenic response and hexose transport. J Biol Chem $1981: 256: 1290-1300$

25) Pinkofsky HB, Rampal AL, Cowden MA and Jung $\mathrm{CY}$ : Cytochalasin $B$ binding proteins in human erythrocyte membranes: Modulation of glucose sensitivity by site interaction and partial solubilization of binding activities. J Biol Chem $1978: 253: 4930$ 4937

26) Blok J, Gibbs EM, Lienhard GE, Slot JW and Geuze HJ : Insulin-induced translocation of glucose transporters from post-Golgi compartments to the plasma membrane of 3T3-L1 adipocytes. J Cell Biol $1988: 106: 69-76$

27) Maguire GA, Docherty K and Hales CN : Sugar transport in rat liver lysosomes: Direct demonstration by using labelled sugars. Biochem J $1983: 212$ :211-218 